\title{
SHORT COMMUNICATION A New Grain Boundary Scattering Model for Metals
}

\author{
F. WARKUSZ \\ Institute of Physics, Wroclaw Technical University, Wybrzeze Wyspianskiego 27, \\ 50-370 Wroclaw, Poland
}

(Received November 29, 1977; in final form April 12, 1978)

The electrical conductivity of metal films is markedly influenced by the grain structure of the material used..$^{1-7}$

In the present paper, an electron grain-boundary scattering function similar to the one obtained by Mayadas and Shatzkes ${ }^{7,8}$ is derived in terms of Cottey's electron mean free path model and method. ${ }^{9}$

The grain are assumed to have equal diameters $D$ separated by grain boundaries $g$ which are assumed to be perpendicular to the film planes and markedly contribute to electron scattering (Figure 1). If an electric field is applied, the electrons approach the grain boundaries at angle $\phi=(\pi / 2)-\theta$ (where $0<\theta<\pi$ ). The coefficient of electron transmission through the grain boundary is $r$, and $0 \leqslant r \leqslant 1$. If no electrons are passing through the grain boundary, then $r=0$. If all electrons cross the grain boundary, then $r=1$. The probability of electrons travelling through $n$ grain boundaries is $P$ and decreases as the number of grain boundaries increases.

Hence

$$
P=r^{n} \text {. }
$$

It is assumed (after Cottey) that $P$ varies proportionally to the travelling distance $l$

$$
-d P=\frac{P}{\lambda_{0}} d l
$$

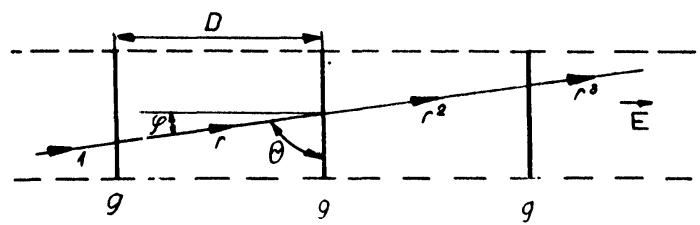

FIGURE 1 Model of the grain structure. where $\lambda_{D}$ is the mean free path associated with the influence of the conduction electrons on the grain boundaries.

After integration of Eq. (2) and considering that $P_{0}=1$ we obtain

$$
P=\exp \left(-l / \lambda_{D}\right)
$$

where $l=n D / \sin \theta$. Combining Eq. (3) and Eq. (1) gives

$$
\lambda_{D}(\theta)=\frac{D}{\sin \theta \ln (1 / r)}
$$

If grain boundary scattering and background scattering are assumed independent, then the mean free path for the resultant scattering becomes

$$
\frac{1}{\lambda(\theta)}=\frac{1}{\lambda_{0}}+\frac{1}{\lambda_{D}(\theta)}
$$

where $\lambda_{0}$ is the background mean free path.

The current density is given by ${ }^{9}$

$$
J=e \int V_{x} f_{1}\left(\theta_{1} \Phi\right) d^{3} p
$$

where $e$ is the electron charge, $V_{x}$ denotes the $\mathrm{x}$ component of electron velocity, $f_{1}$ stands for the deviation from the equilibrium function of Fermi distribution.

Thus the formula for the electrical conductivity of the film becomes ${ }^{9}$

$$
\sigma=\frac{J}{E}=\frac{2 \pi e^{2} P_{F}^{2}}{h^{3}} \sin ^{3} \theta \lambda(\theta) d \theta
$$

where $p_{F}$ is the Fermi momentum of the electron, and $h$ is Planck's constant. 
Combining Eq. (5) and Eq. (7) and considering Eq. (4) we obtain

$$
\frac{\sigma_{g}}{\sigma_{0}}=\frac{3}{4} \frac{\sin ^{3} \theta}{1+\frac{\lambda_{0} \ln 1 / r}{D} \sin \theta} d \theta \equiv F(\nu)
$$

The integration of Eq. (8) gives

$$
\begin{aligned}
F_{2}(\nu)= & \frac{3}{2} \nu \frac{\pi}{4}-\nu+{ }_{2} \nu^{2}+\frac{\nu^{3}}{\sqrt{1-\nu^{2}}} \ln \frac{\nu}{1+\sqrt{1-\nu^{2}}} \\
& \text { for } \nu<1 \\
= & \frac{3 \pi}{8} \nu-\frac{3}{2} \nu^{2}+\frac{3 \pi}{4} \nu^{3}+\frac{3}{2} \nu^{4} \ln \nu+\frac{3}{8} \nu^{6}+\cdots
\end{aligned}
$$

$$
\begin{aligned}
F_{2}(\nu)= & \frac{3}{2} \nu \frac{\pi}{4}-\nu+\frac{\pi}{2} \nu^{2}-\frac{\nu^{3}}{\sqrt{\nu^{2}-1}} \arcsin \sqrt{1-\frac{1}{\nu^{2}}} \\
& \text { for } \nu>1 \\
= & 1-\frac{9 \pi}{32 \nu}+\frac{4}{5 \nu^{2}}-\frac{15}{64 \nu^{3}}+\cdots
\end{aligned}
$$$$
F_{3}(\nu)=\frac{3}{8}(3 \pi-8)=0.5342 \text { for } \nu=1
$$

where

$$
\nu=\frac{D}{\lambda_{0} \ln 1 / r}
$$

The grain boundary resistivity $\rho_{g}$, i.e. the resistivity of an infinitely thick polycrystalline film, is given by

$$
\frac{\rho_{g}}{\rho_{0}}=\frac{1}{F(\nu)}
$$

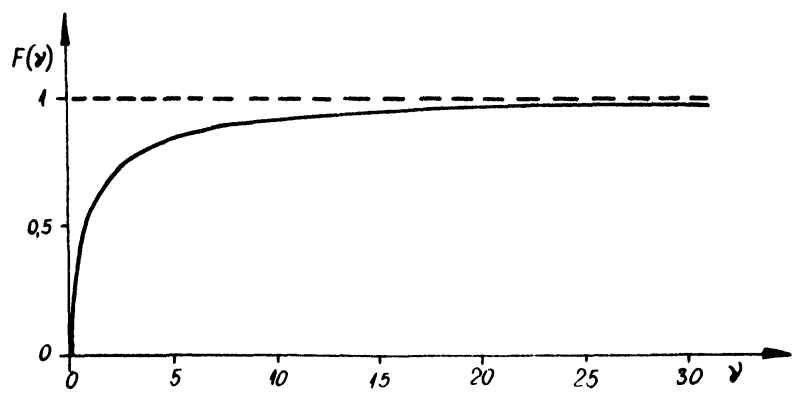

FIGURE $2 \quad F(\nu)$ versus $\nu$ curves (plotted using Eq. (9) and Eq. (10)).

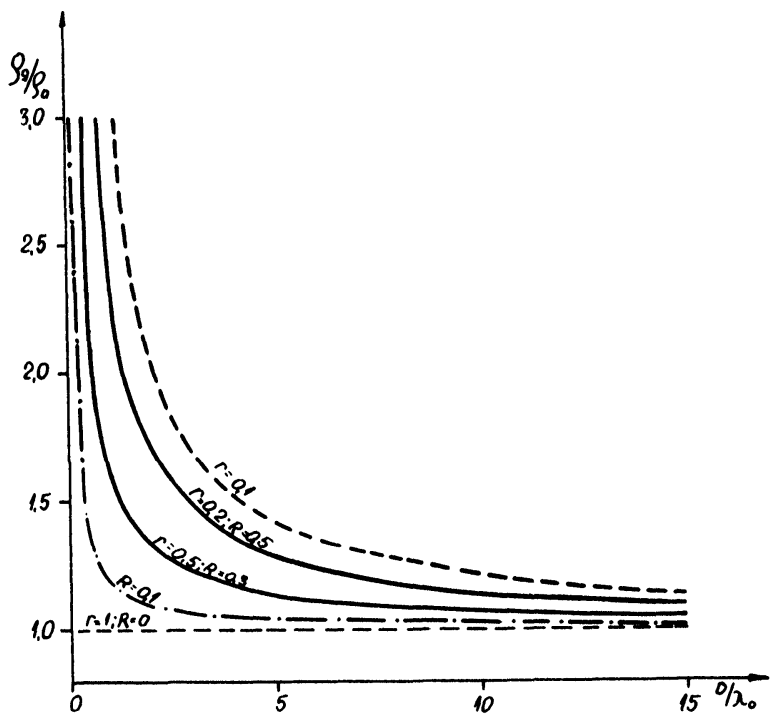

FIGURE $3 \rho_{g} / \rho_{0}$ versus $D / \lambda_{0}$ curves (plotted using Eq. (9), Eq. (10) and Eq. (11) for $r=0.1,0.2$ and 0.5 and Eq. (13) for $R=0.1,0.3$ and 0.5 .

where $\rho_{0}$ is the bulk resistivity, and $F(\nu)$ denotes the function expressed in Eq. (8). From Figure 2 it is evident that if $\nu \rightarrow \infty$ then $F(\nu) \rightarrow 1$, and if $\nu \rightarrow 0$, then $\mathrm{F}(\nu) \rightarrow 0$. Considering Eqs. (12), (9), (10) and (11), the $\rho_{g} / \rho_{0}$ versus $D / \lambda_{0}$ curves were plotted (Figure 3 ) for $r=0.1,0.2$ and 0.5 . It follows that:

1) Resistivity $\rho_{g}$ is significantly higher than $\rho_{0}$ when $D<\lambda_{0}$.

2) If $D \gg \lambda_{0}$, then $\rho_{g}$ approaches $\rho_{0}$.

3) Resistivity $\rho_{g}$ depends on the coefficient $r$, and decreases as $r$ increases.

Functions $F_{1}(\nu)$ and $F_{2}(\nu)$ are similar to the function $G(\alpha)^{7,8}$

$G(\alpha)=1-\frac{3}{2} \alpha+3 \alpha^{2}-3 \alpha^{3} \ln \left(1+\frac{1}{\alpha}\right)$

where $\alpha=\lambda_{0} R / D(1-R)(R$ is the grain boundary electron reflection coefficient). If $R=0$ then $\rho_{g}=\rho_{0}$; if $R \rightarrow 1$, then $\rho_{g} \rightarrow \infty$.

Comparing Eqs. (9), (10) and (13) gives two alternative expressions: $\nu=0.62 / \alpha$ or $R /(1-R)=$ $0.62 \ln (1 / r)$. If, for example, $r=0.2$, then $R=0.5$, and if $r=0.5$, then $R=0.3$. This is evident in Figure 3 (either of the full lines represents both our results and the Mayadas-Shatzkes functions).

Eq. (12) is true for polycrystalline thick films alone because the resistivity of thin films is additionally influenced by the external size effect..$^{3,4,10}$ 
The grain diameter dependence of resistivity for $\mathrm{Al}$ films was studied experimentally, and the results obtained are reported by Dobierzewska-Mozrzymas and Warkusz. ${ }^{4}$

\section{REFERENCES}

1. P. V. Andrews, M. B. West and C. R. Robeson, Phil. Mag., 19, 887 (1969).

2. V. P. Nagpal and V. P. Duggal, Thin Solid Films, 9, 313 (1972).
3. P. Wissmann, Thin Solid Films, 5, 329 (1970).

4. E. Dobierzewska-Mozrzymas and F. Warkusz, Thin Solid Films, 43, 267 (1977).

5. P. G. Wassilev, Bulg. J. Phys., 3, 68 (1976).

6. R. A. Brown, J. Phys. F.: Metal Phys., 7, 1477 (1977).

7. A. F. Mayadas and M. Shatzkes, Phys. Rev. B, 1, 1382 (1970).

8. A. F. Mayadas, M. Shatzkes and J. F. Janak, Appl. Phys. Lett., 14, 345 (1969).

9. A. A. Cottey, Thin Solid Films, 1, 297 (1967).

10. F. Warkusz, Electrocom. Sci. and Tech. (1978), in press. 

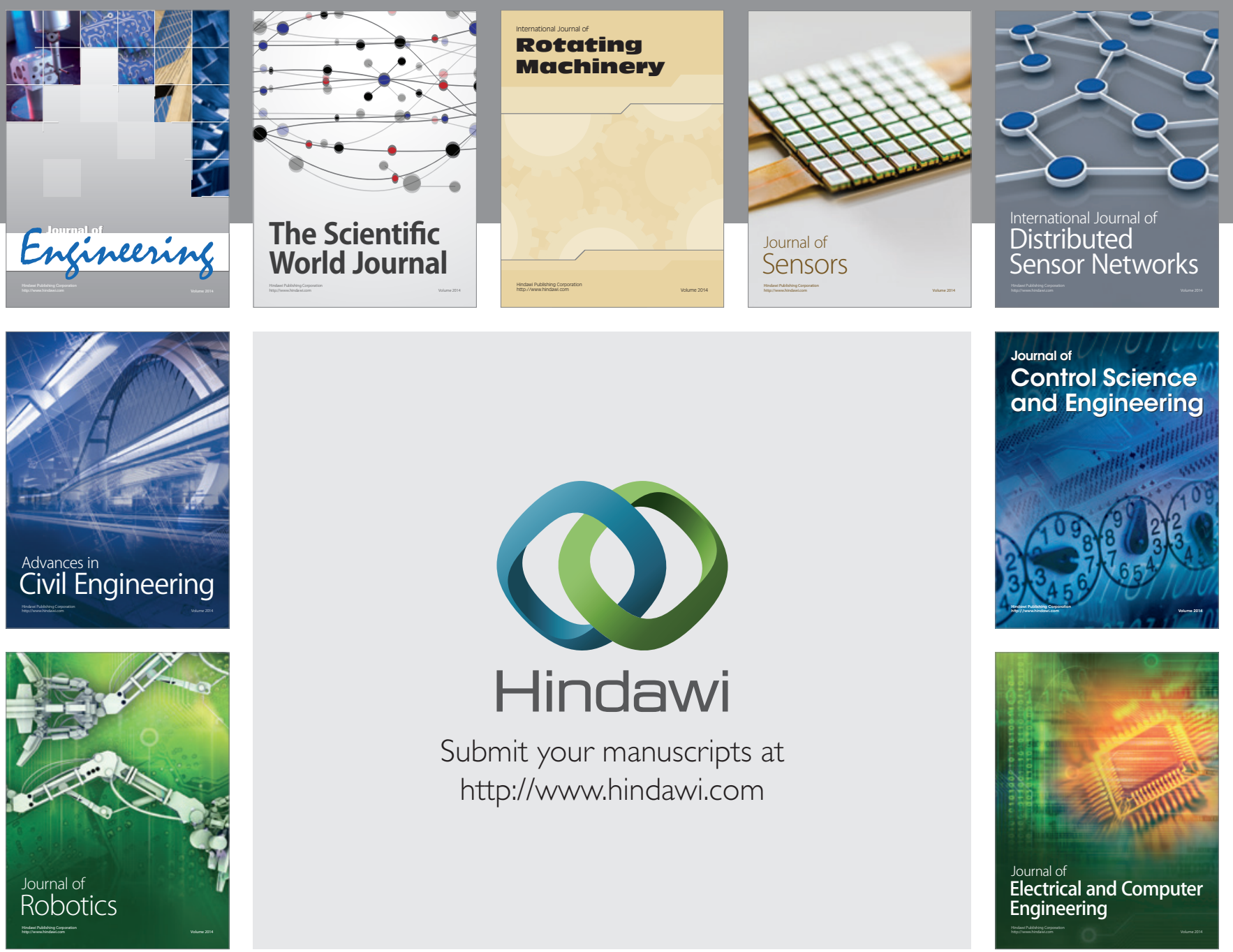

Submit your manuscripts at

http://www.hindawi.com
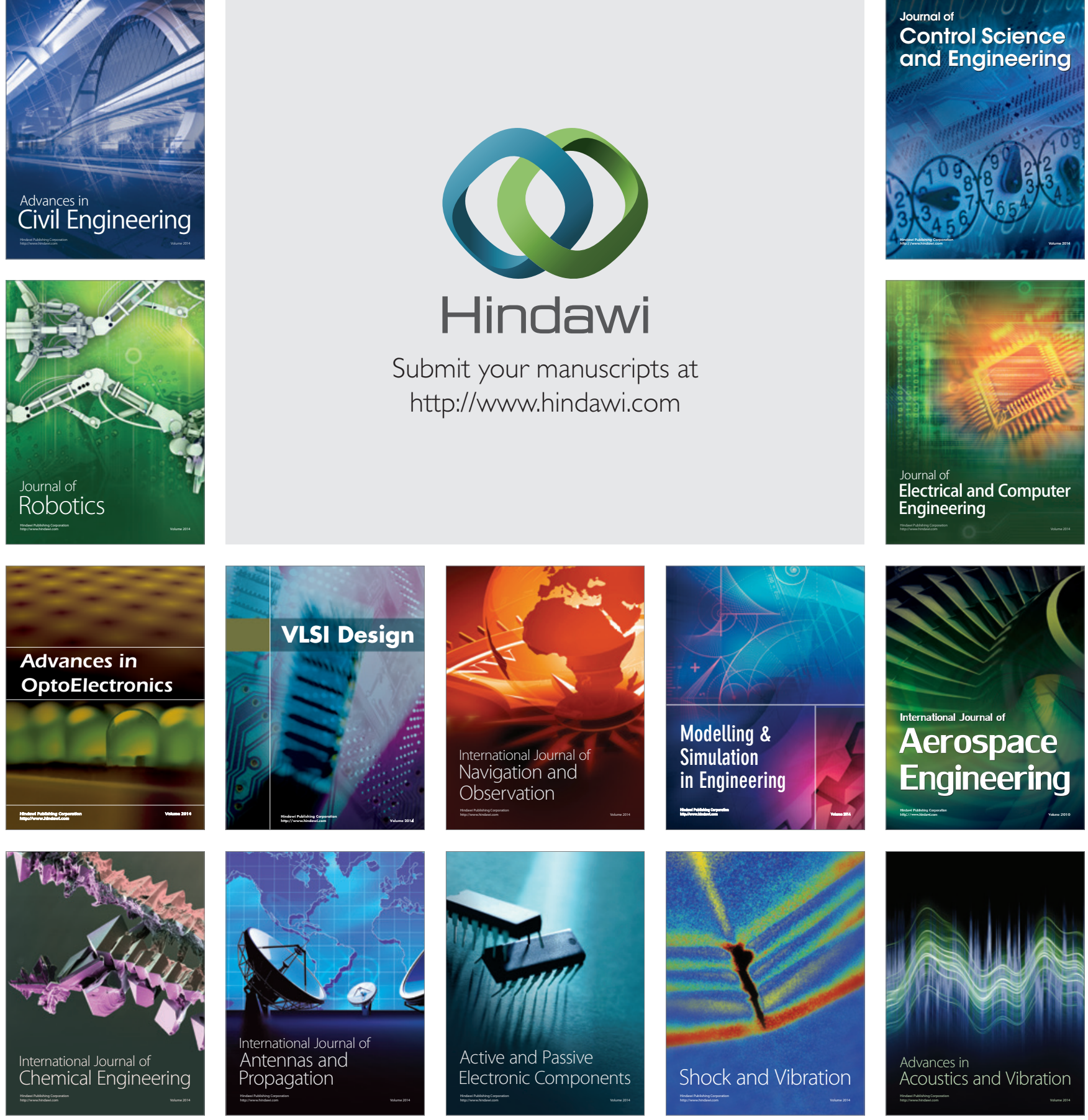